\title{
Caracterização morfológica e luminescente de nanopartículas de aluminato de zinco dopadas com $\mathrm{Eu}^{3+}$
}

\section{(Luminescence and morphology of zinc aluminate doped with Eu $\boldsymbol{u}^{3+}$ nanoparticles)}

\author{
B. S. Barros ${ }^{1}$, P. S. Melo , L. Gama, S. Alves-Jr', E. Fagury-Neto ${ }^{3}$, \\ R. H. G. A. Kiminami ${ }^{3}$, A. C. F. M. Costa ${ }^{1}$ \\ ${ }^{I}$ Departamento de Engenharia de Materiais, Universidade Federal de Campina Grande, Campina Grande, PB \\ ${ }^{2}$ Laboratório de Terras-Raras - BSTR, Departamento de Engenharia de Materiais, Química Fundamental, \\ Universidade Federal de Pernambuco, Recife, PE \\ ${ }^{3}$ Departamento de Engenharia de Materiais, Universidade Federal de S. Carlos \\ Rod. Washington Luiz, km 235, C.P. 676, S. Carlos, SP 13565-905
}

\begin{abstract}
Resumo
Este trabalho reporta a obtenção de nanopartículas de aluminato de zinco dopado com $\mathrm{Eu}^{3+}$, por meio do método de síntese por reação de combustão. Foram preparadas quatro amostras com diferentes concentrações de dopante, para avaliar o efeito do teor de $\mathrm{Eu}^{3+}$ na luminescência dos pós obtidos. Os resultados de difração de raios $\mathrm{X}$ confirmaram a formação da fase espinélio $\mathrm{ZnAl}_{2} \mathrm{O}_{4} \mathrm{e}$ também traços de fases secundárias identificadas como $\mathrm{EuAlO}_{3}$ e $\mathrm{ZnO}$, em quantidade diretamente proporcional ao teor de dopante das amostras. O espectro de emissão foi excitado em $265 \mathrm{~nm}\left(\lambda_{\max }\right)$, o qual apresentou picos característicos do íon Eu ${ }^{3+}$ localizados em torno de 578, 591613,653 e $703 \mathrm{~nm}$. Os resultados obtidos por microscopia eletrônica de varredura apresentam aglomerados em forma de placas irregulares formadas por nanopartículas com pontos dispersos de fase secundaria na superfície. Baseado nos resultados verificou-se a dependência da intensidade de luminescência com o teor e também com a forma de incorporação do íon $\mathrm{Eu}^{3+}$, inserido na rede hospedeira, adsorvido na superfície de nanopartículas ou formando uma segunda fase.

Palavras-chave: nanopartículas, luminescência, reação de combustão.
\end{abstract}

\begin{abstract}
This paper reports how zinc aluminate nanoparticles doped with $E u^{3+}$ ions were obtained by the method of combustion reaction synthesis. Four samples were prepared with different amounts of dopant to evaluate the effect of the concentration of Eu ${ }^{3+}$ ions on the powders' luminescence. X-ray diffraction results confirmed the formation of the spinel phase, as well as secondary phases identified as $\mathrm{EuAlO}_{3}$ and $\mathrm{ZnO}$, in amounts directly proportional to the content of dopant in the samples. An excitation wavelength of $265 \mathrm{~nm}$ was determined based on the excitation spectrum. This wavelength was used to obtain the emission spectrum, which revealed the presence of peaks characteristic of Eu ${ }^{3+}$ ions located at around 578, 591 613, 653 and $703 \mathrm{~nm}$. SEM analysis indicated that the powder's morphology consisted of irregular plate-shaped agglomerates composed of nanoparticles with scattered points of secondary phase on their surface. These results indicate how the intensity of luminescence is dependent on the Eu ${ }^{3+}$ ion content and on how these ions are incorporated, i.e., inserted into the host lattice, adsorbed on the surface, or forming a secondary phase.
\end{abstract}

Keywords: nanoparticles, luminescence, combustion reaction.

\section{INTRODUÇÃO}

O aluminato de zinco é um óxido com estrutura do tipo espinélio normal $\mathrm{AB}_{2} \mathrm{O}_{4}$, que consiste de um arranjo de empacotamento fechado cfc de átomos de oxigênio com grupo espacial de simetria $F d 3 m$, apresentando sítios cristalográficos não equivalentes tetraédricos $A$ e octaédricos $B$, onde $A$ e $B$ são os sítios de rede ocupados pelos íons bivalentes e trivalentes, respectivamente [1-3]. O aluminato de zinco é um semicondutor que apresenta um gap de energia em torno de 3,8 eV [4], o que indica a sua transparência para luz com comprimento de onda superior a $320 \mathrm{~nm}$. Esta característica o torna bastante atraente como rede hospedeira para íons capazes de gerarem luminescência, tais como, íons de metais de transição e íons de terras raras. Atualmente, vários autores têm reportado a obtenção de pós de aluminato de zinco dopado com íons terras raras para uso como fósforos de alta eficiência em displays tipo FED (Field Emission Display) e PDP (Plasma Display Panels) [5-7]. Materiais luminescentes a base destes óxidos são preferíveis em relação aos materiais à base de enxofre, como por exemplo ZnS dopado com íons de metais de transição ou terras raras, 
devido aos óxidos serem quimicamente inertes [7].

Características morfológicas e estruturais também são fatores importantes na obtenção de materiais luminescentes. Segundo Bhagrava e Galagher [12], nanopartículas semicondutoras dopadas com lantanídeos apresentam significativa elevação de transições radiativas e o decréscimo no tamanho das partículas implica diretamente em um aumento da eficiência de emissão de pós-fosforescentes $[6,12]$, o que justifica o crescente aumento nos estudos sobre lantanídeos dopando materiais nanocristalinos, com partículas de diâmetro inferior a $100 \mathrm{~nm}$.

A incorporação ou dopagem dos íons lantanídeos em matrizes sólidas semicondutoras, proporcionam a emissão de luminescência devido ás transições entre níveis internos $4 \mathrm{f}$ e é observada em vários materiais semicondutores. Os elétrons $\mathrm{f}^{\mathrm{h}}$, nestes íons, encontram-se protegidos de interações com o meio. Esta proteção dos íons lantanídeos é atribuída ao efeito de blindagem conferido pelos orbitais $5 s$ e $5 p$ mais externos e já preenchidos. Este efeito de blindagem é responsável por muitas propriedades químicas e físicas na maioria dos lantanídeos [13]. As transições mais interessantes, resultantes da emissão de luz no visível (em bandas relativamente finas, comparado aos metais de transição), são aquelas intra $4 f(4 f \rightarrow 4 f)$ [13].

Diversos métodos de síntese têm sido utilizados para a obtenção de pós com partículas nanométricas de aluminato de zinco, dentre os quais podemos citar a síntese hidrotérmica $[8,9]$, coprecipitação [8] e sol-gel [10,11]. Entre os métodos químicos, a síntese por reação de combustão destaca-se como uma técnica promissora para a preparação de pós de $\mathrm{ZnAl}_{2} \mathrm{O}_{4}$ : $\mathrm{Eu}^{3+}$, com elevado grau de pureza e pequeno tamanho de partículas. O método de combustão é auto-sustentável após o inicio da reação e atinge altas temperaturas, que garantem a cristalização e formação de pós em curto período de tempo, com liberação de grande quantidade de gases, o que tende a minimizar o estado de aglomeração das partículas que se formam. Sendo assim considerado simples, o método de combustão não envolve muitas etapas e produz pós com elevado grau de pureza, homogeneidade química e quase sempre em escala nanométrica [14-15]. Este material apresenta interessantes características de emissão, que podem ser relacionadas com a presença de poros e também ao tamanho das partículas [16].

Com base nestes aspectos, o presente trabalho tem como objetivo a preparação via reação de combustão, caracterização morfológica e luminescente de nanoparticulas aluminato de zinco dopadas com íons $\mathrm{Eu}^{3+}$.

\section{MATERIAIS E MÉTODOS}

Neste estudo, foram preparados pós de $\mathrm{ZnAl}_{2-\mathrm{x}} \mathrm{Eu}_{\mathrm{x}} \mathrm{O}_{4}$, com $\mathrm{x}$ assumindo os valores $0,025,0,05,0,075$ e 0,1 mol por meio do método de síntese por reação de combustão. Este método envolve uma mistura contendo os íons metálicos, como reagentes oxidantes (nitratos e óxidos), e um combustível (uréia), como agente redutor. Os materiais utilizados para a mistura foram nitrato de alumínio $\mathrm{Al}\left(\mathrm{NO}_{3}\right)_{3} 9 \mathrm{H}_{2} \mathrm{O}$
(Merck), nitrato de zinco $\mathrm{Zn}\left(\mathrm{NO}_{3}\right)_{2} 6 \mathrm{H}_{2} \mathrm{O}$ (Merck), óxido de európio $\mathrm{Eu}_{2} \mathrm{O}_{3}$ (Aldrich) e uréia $\mathrm{CO}\left(\mathrm{NH}_{2}\right)_{2}$ (Synth), todos de elevada pureza, atestada pelos respectivos fabricantes. As proporções de cada reagente e combustível foram calculadas a partir das valências dos elementos reativos de modo a favorecer a relação entre as quantidades de oxidante e de combustível, com base na estequiometria preestabelecida dos íons metálicos para formar a fase de interesse, usandose os conceitos da química dos propelentes [17]. Carbono, hidrogênio, alumínio, zinco e európio foram considerados como elementos redutores, com valências respectivas de 4+, $1+, 3+, 2+$ e $3+$. O oxigênio foi considerado como agente oxidante, tendo valência 2-. A valência considerada para o nitrogênio foi 0 (zero). As soluções preparadas de acordo com a composição estequiométrica foram misturadas em um becker pirex e submetidas ao aquecimento diretamente em uma placa quente à temperatura aproximada de $480{ }^{\circ} \mathrm{C}$ até a auto-ignição ocorrer. $\mathrm{O}$ pó resultante na estrutura de flocos porosos e de coloração branca foi então transferido para uma mufla pré-aquecida a $500{ }^{\circ} \mathrm{C}$, na qual permaneceu por 15 minutos para eliminação de voláteis (advindos da decomposição da uréia e dos nitratos) que poderiam estar presentes, sendo então peneirado em malha $325(0,044 \mu \mathrm{m})$.

Os pós-resultantes da reação de combustão foram caracterizados por meio de difração de raios X (DRX), para identificação das fases presentes, medidas de parâmetros de rede e do tamanho médio de cristalito pelo alargamento do pico de difração de maior intensidade da fase majoritária, usando a formula de Scherrer [18]. Foi usado difratômetro de raios X Shimadzu (modelo XRD-6000, radiação $\mathrm{Cu} \mathrm{K \alpha}$ ) que opera com tubo de alvo de cobre a uma voltagem de 40 $\mathrm{kV}$ com $30 \mathrm{~mA}$ de corrente. Os difratogramas foram obtidos na faixa $2 \theta$ de 10 a 80 graus em passos de $0,03^{\circ}$ e tempo de passo de $1 \mathrm{~s}$ na temperatura ambiente de $25^{\circ} \mathrm{C}$. A morfologia dos pós e o tamanho médio de aglomerados foram observados através de microscopia eletrônica de varredura (MEV), em equipamento Philips, modelo XL30 - FEG, onde também foram realizadas as análises por energia dispersiva (EDS), para determinar e quantificar a presença do íon $\mathrm{Eu}^{3+}$ na rede hospedeira. As medidas de fotoluminescência foram realizadas através de espectroscopia de excitação e emissão, a partir de amostras sólidas na forma de pó e em temperatura ambiente, usando-se o ISS K2 Multifrequency Phase Fluorimeter.

\section{RESULTADOS E DISCUSSÃO}

Parâmetros como o tempo e a cor da chama foram observados durante o desenvolvimento experimental com o intuito de avaliar o processo de síntese por reação de combustão e os resultados estão apresentados na Tabela I.

A temperatura e o tempo de reação são dois fatores decisivos no controle da transformação de fase durante o processo de síntese por combustão [19]. Porém estes dois fatores podem ser controlados por meio do controle adequado das condições de aquecimento (mufla, placa-mufla ou placa), 
Tabela I - Características da reação de combustão.

[Table I-Characteristics of the combustion reaction.]

\begin{tabular}{ccl}
\hline $\begin{array}{c}\text { Teor de Eu} \\
(\mathrm{mol})\end{array}$ & $\begin{array}{c}\text { Tempo de } \\
\text { Chama (s) }\end{array}$ & $\begin{array}{c}\text { Cor da } \\
\text { chama }\end{array}$ \\
\hline 0,025 & 9 & Amarela \\
\hline 0,050 & 16 & Amarela \\
\hline 0,075 & 17 & Amarela \\
\hline 0,100 & 15 & Amarela \\
\hline
\end{tabular}

do tipo de precursor utilizados (carbonatos, nitratos ou acetatos), do tipo de combustível (uréia, glicina ou diidrazina maléica) e pelas condições de realização da própria síntese (becker, cadinho de sílica vítrea ou cadinho de porcelana) [20]. Porém a temperatura da chama de combustão varia de material para material e é uma característica intrínseca de cada sistema. O controle de ambos os parâmetros é importante, visto que as características finais dos pós, como tamanho de partícula e grau de aglomeração dependem diretamente destes dois parâmetros.

A Tabela I apresenta os valores de tempo de chama de combustão e a cor da chama para os sistemas dopados com európio. Pelos dados apresentados na Tabela I podemos observar que, com exceção da amostra dopada com 0,025 mol de íon $\mathrm{Eu}^{3+}$, todas as outras apresentaram características de reação bem semelhantes. O baixo tempo de chama de combustão determinado para a amostra com $0,025 \mathrm{~mol}$ de dopante foi provavelmente uma conseqüência da alta umidade adquirida pelo reagente nitrato de zinco utilizado, devido as condições climáticas (alta umidade relativa do ar) durante a execução da pesagem dos precursores, o que alterou a estequiometria do sistema. $\mathrm{O}$ aumento na concentração de íon európio não alterou a cor da chama, o que indica, que as temperaturas de chama da combustão máximas foram próximas para todas as composições.

A Fig. 1 apresenta os difratogramas de raios $\mathrm{X}$ dos pós da fase espinélio $\mathrm{ZnAl}_{2} \mathrm{O}_{4}$ dopada com íons $\mathrm{Eu}^{3+}$ obtidos pela síntese por reação de combustão. Em todas as composições analisadas foram identificados picos de difração bem definidos e com elevado grau de cristalização, característicos da fase majoritária com estrutura tipo espinélio (JCPDS \# 82-1043) e também picos correspondentes a pequenas quantidades das fases secundárias $\mathrm{EuAlO}_{3}$ (JCPDS \# 09-0084) e ZnO (JCPDS \# 36-1451). Verifica-se que a quantidade de fase secundaria é diretamente proporcional à concentração de íon dopante utilizada durante a síntese. Isto pode ser comprovado pelo aumento nas intensidades dos picos de difração das fases secundárias nas amostras com maior teor de $\mathrm{Eu}^{3+}$. Para a amostra dopada com 0,025 mol de íons $\mathrm{Eu}^{3+}$ observamos uma fuga da tendência de formação de fases secundárias. Nesta amostra, podemos perceber a presença de quantidade de segundas fases superior a das amostras dopadas com 0,05 , 0,075 e 0,1 moles, isto provavelmente devido ao menor tempo de chama de combustão provocado pela perda de estequiometria.

A Tabela II apresenta os tamanhos de cristalito calculados com base na largura de meia altura do pico de difração de

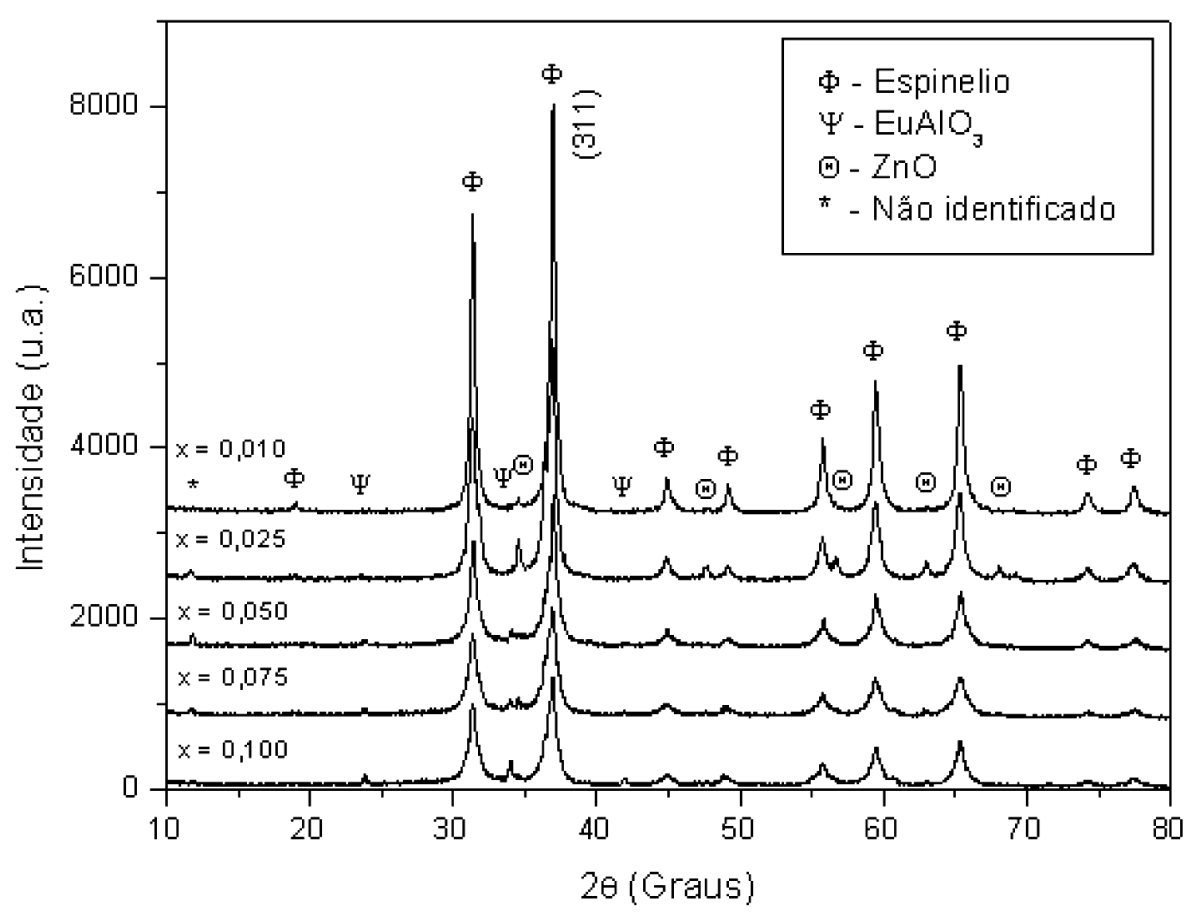

Figura 1: Difratogramas de raios $\mathrm{X}$ dos pós de $\mathrm{ZnAl}_{2} \mathrm{O}_{4}: \mathrm{Eu}^{+}$obtidos por reação de combustão. [Figure 1: X-ray powder diffraction pattern of $\mathrm{ZnAl}_{2} \mathrm{O}_{4}: \mathrm{Eu}^{+}$obtained by combustion reaction.] 
maior intensidade da fase majoritária, correspondente ao plano (311), por meio da formula de Scherrer [18]. Todas as amostras obtidas apresentaram tamanho de cristalito inferior a $20 \mathrm{~nm}$, o que comprova a característica nanométrica dos pós obtidos.
Os parâmetros de rede calculados com o software PowderX [21] a partir dos difratogramas de raios X, são apresentados na Tabela II. Observa-se uma pequena variação dos valores obtidos, relacionada com a concentração de dopante utilizada e também com as características da reação. Esta variação nos
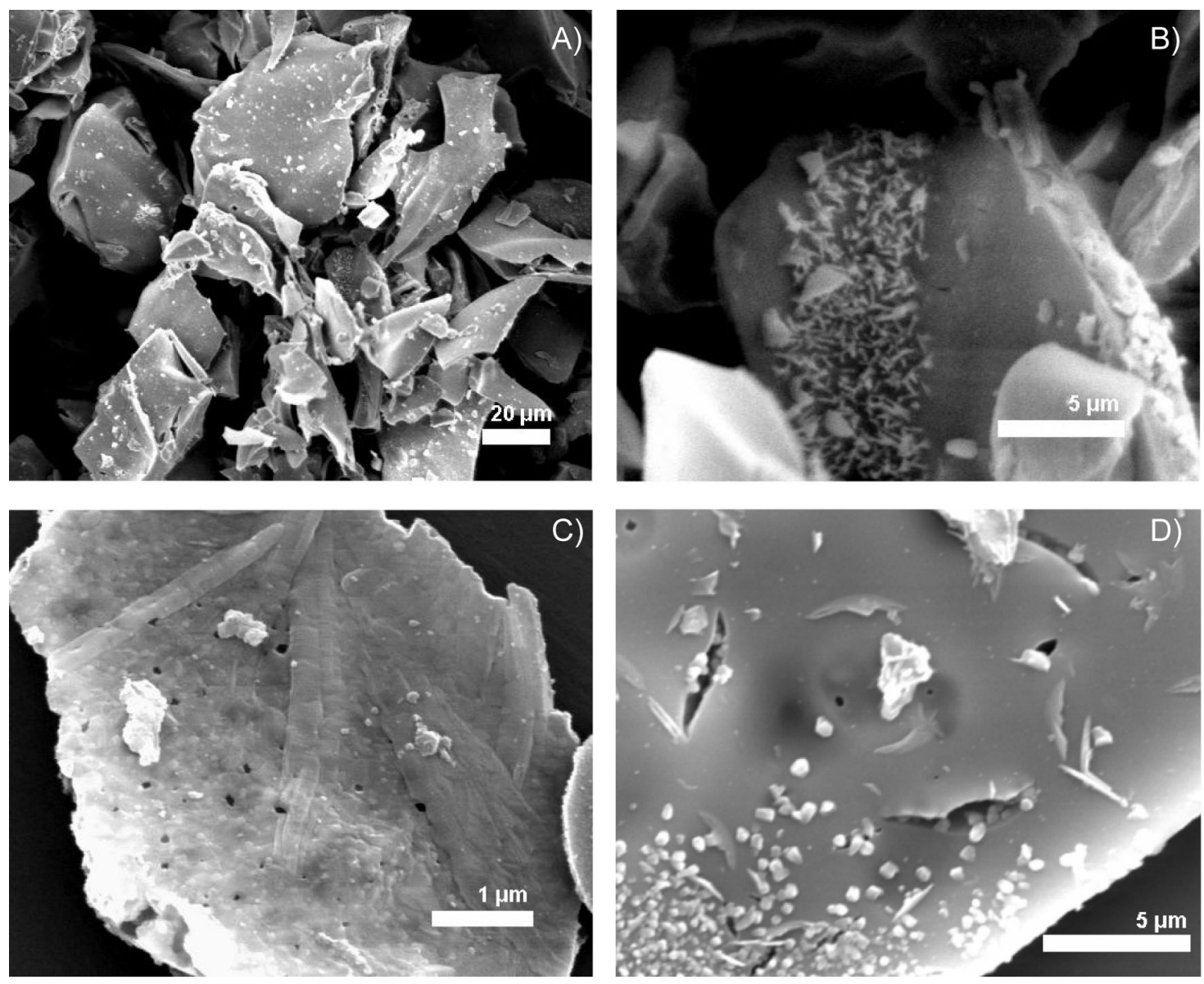

Figura 2: Aspectos morfológicos do pó de $\mathrm{ZnAl}_{2} \mathrm{O}_{4}: \mathrm{Eu}^{3+}$ obtido pela reação de combustão. (a) 600x, (b) 4.500x, (c) $30.000 \mathrm{x}$, (d) 7.000x.

[Figure 2: Morphologic aspects of the powder of ZnAl2O4:Eu $u^{3+}$ obtained by the combustion reaction. (a) x600, (b) $x 4500,(c) \times 30000,(d) \times 7000$.

Tabela II - Teor de dopante da amostra, grau de cristalinidade do pó resultante, tamanho de cristalito e parâmetros de rede da fase majoritária (espinélio).

[Table II - Dopant concentration of the sample, crystallinity degree of the resulting powder, crystallite size and lattice parameter of the majority phase (spinel).]

\begin{tabular}{cccc}
\hline $\begin{array}{c}\text { Teor de Eu } \\
(\mathrm{mol})\end{array}$ & $\begin{array}{c}\text { Grau de } \\
\text { cristalinidade }(\%)\end{array}$ & $\begin{array}{c}\text { Tamanho de } \\
\text { cristalito }(\mathrm{nm})\end{array}$ & $\begin{array}{c}\text { Parâmetros de } \\
\text { rede }<\mathrm{a}=\mathrm{b}=\mathrm{c}>(\AA)\end{array}$ \\
\hline 0,025 & 58,6 & 16 & 8,083 \\
\hline 0,050 & 50,0 & 18 & 8,086 \\
\hline 0,075 & 48,4 & 14 & 8,084 \\
\hline 0,100 & 50,3 & 10 & 8,088 \\
\hline
\end{tabular}




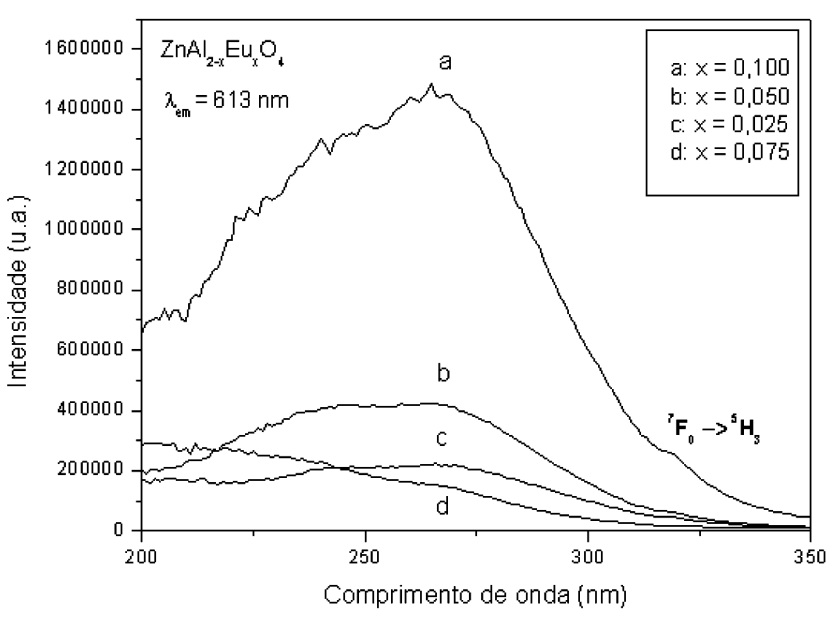

Figura 3: Espectro de excitação do pó de $\mathrm{ZnAl}_{2} \mathrm{O}_{4}: \mathrm{Eu}^{3+}$.

[Figure 3: Excitation spectrum of $\mathrm{ZnAl}_{2} \mathrm{O}_{4}: \mathrm{Eu}^{3+}$ powder.]

parâmetros de rede é provavelmente uma conseqüência da formação de vacâncias dentro da rede hospedeira, motivada pela entrada do íon $\mathrm{Eu}^{3+}$. Este íon de raio iônico elevado (0,95 ̊̊), deverá ocupar preferencialmente sítios octaédricos, normalmente ocupados por íons $\mathrm{Al}^{3+}$, de pequeno raio iônico $(0,5 \AA)$, conseqüentemente causando uma pequena distorção na rede o que pode estar provocando a expulsão de íons $\mathrm{Zn}^{2+}$ em sítios tetraédricos próximos. Isto explica o aumento da quantidade de fase secundária $(\mathrm{ZnO})$ para teores de dopante mais elevados. Porém, apesar das variações observadas, os parâmetros de rede calculados mostraram boa concordância com os valores encontrados na literatura, $\mathrm{a}=\mathrm{b}=\mathrm{c}=8,088 \AA$ (JCPDS \# 82-1043), principalmente na amostra dopada com 0,1 mol devido à formação em maior quantidade da fase $\mathrm{EuAlO}_{3}$, diminuindo assim a quantidade de íons $\mathrm{Eu}^{3+}$ dentro da rede hospedeira $\mathrm{ZnAl}_{2} \mathrm{O}_{4}$ e provocando uma menor distorção da mesma. Strek e colaboradores [6], reportando a obtenção de pós nanoestruturados de $\mathrm{ZnAl}_{2} \mathrm{O}_{4}$ através de síntese hidrotérmica observaram que os íons $\mathrm{Eu}^{3+}$ também podem se encontrar adsorvidos na superfície de nanoparticulas devido à alta porosidade do espinélio.

$\mathrm{O}$ aspecto morfológico dos pós resultantes da síntese por reação de combustão observado por microscopia eletrônica de varredura pode ser visto na Fig. 2. Através destas micrografias podemos observar a formação de aglomerados duros com tamanho variando entre 5 e $40 \mu \mathrm{m}$. Estes aglomerados apresentam-se com morfologia irregular na forma de placas, da fase majoritária identificada através de difração de raios $\mathrm{X}$ como $\mathrm{ZnAl}_{2} \mathrm{O}_{4}$ e são constituídos de pequenas partículas em escala nanométrica. Sobre a superfície dos aglomerados da fase $\mathrm{ZnAl}_{2} \mathrm{O}_{4}$ foi possível observar pequenos aglomerados e pequenas partículas de fases secundárias também identificadas por difração de raios X (Figs. 4a e 4b). A formação de fases secundárias e a deposição destas na superfície dos aglomerados da fase majoritária foram proporcionadas pela baixa solubilidade do európio na matriz hospedeira $\mathrm{ZnAl}_{2} \mathrm{O}_{4}$. A Fig. 4c mostra a formação de poros nos aglomerados devido a rápida liberação de gases pelos produtos da reação [22]. Na Fig. 4d, podemos observar a superfície de um grande aglomerado formado pela fase majoritária (com coloração mais escura) e sobre a mesma, pequenos aglomerados de fase secundária (com coloração mais clara) que estão sendo expelidos para fora do aglomerado da fase majoritária através de fendas provavelmente formadas a partir de poros.

Para que se possa ter uma idéia do que os aglomerados apresentavam em sua constituição, foram realizadas medidas de EDS de duas formas: primeiramente, foi focalizada a superfície lisa que não apresentava outras partículas aglomeradas na superfície (fase majoritária) e depois foram feitas as medidas em fases aglomeradas na superfície (fase secundária). Os resultados obtidos estão apresentados na Tabela III e podem ser interpretados de forma qualitativa, visto que, devido ao pequeno tamanho dos aglomerados, os resultados são afetados pelas concentrações de átomos presentes nas vizinhanças do ponto analisado. Os resultados obtidos mostram que em todas as amostras preparadas, o íon $\mathrm{Eu}^{3+}$ não foi totalmente introduzido na rede hospedeira formada pela fase majoritária $\mathrm{ZnAl}_{2} \mathrm{O}_{4}$. Este foi também encontrado na fase $\mathrm{ZnO}$, como pode ser observado nos dados referentes à amostra dopada com 0,025 mol, onde não existe a presença da fase $\mathrm{EuAlO}_{3}$. Mesmo assim, pode-se observar um teor considerável de európio $(1,15 \%)$, indicando que este íon também pode ter sido introduzido na rede cristalina do $\mathrm{ZnO}$. A grande quantidade de $\mathrm{ZnO}$, desta amostra é confirmada pelo baixo teor de zinco na fase majoritária $(4,96 \%)$.

A Fig. 3 apresenta o espectro de excitação do aluminato de zinco dopado com $\mathrm{Eu}^{3+}$. A excitação foi realizada varrendo-se o espectro de 200 a $350 \mathrm{~nm}$. A banda de excitação observada para a cor vermelha $(613 \mathrm{~nm})$, encontrase centrada em torno de $265 \mathrm{~nm}$. Esta banda é originada por transições de transferência de carga do íon $\mathrm{O}^{2-}$ para o íon $\mathrm{Eu}^{3+}$ [7]. O estado de transferência de cargas é normalmente o mecanismo de excitação mais intenso e ocorre geralmente

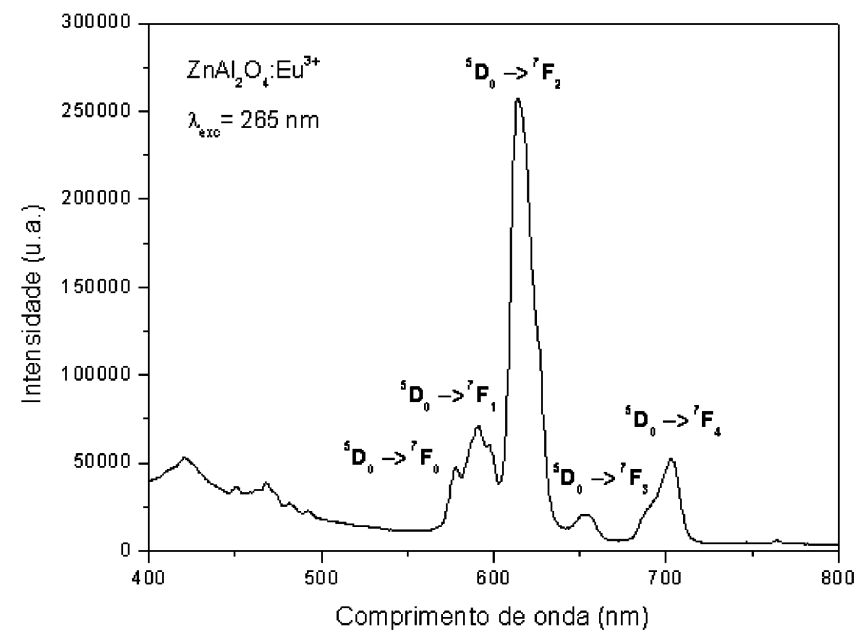

Figura 4: Espectro de emissão do $\mathrm{ZnAl}_{2} \mathrm{O}_{4}$ dopado com $\mathrm{Eu}^{3+}$. [Figure 4: Emission spectrum of $\mathrm{ZnAl}_{2} \mathrm{O}_{4}$ doped with $\mathrm{Eu}^{3+}$.] 
Tabela III - Percentagem atômica de oxigênio, zinco, alumínio e európio das fases primária e secundária resultantes da reação de combustão, determinadas por EDS para os diferentes teores de dopante em alguns agregados.

[Table III - Atomic percents of oxygen, zinc, aluminum and europium of the primary phases and secondary resultants of the combustion reaction, determined by EDS for the different dopant concentration in some agglomerates.]

\begin{tabular}{lcccccccc}
\hline Teor de $\mathrm{Eu}^{3+}$ & \multicolumn{3}{c}{ Fase Primária (\%) } & \multicolumn{4}{c}{ Fase Secundária (\%) } \\
\cline { 2 - 9 }$<\mathrm{X}>(\mathrm{mol})$ & $\mathrm{O}$ & $\mathrm{Zn}$ & $\mathrm{Al}$ & $\mathrm{Eu}$ & $\mathrm{O}$ & $\mathrm{Zn}$ & $\mathrm{Al}$ & $\mathrm{Eu}$ \\
\hline 0,025 & 60,39 & 4,96 & 33,97 & 0,68 & 61,15 & 7,90 & 29,79 & 1,15 \\
\hline 0,050 & 55,01 & 8,08 & 36,32 & 0,59 & 61,45 & 10,59 & 27,13 & 0,83 \\
\hline 0,075 & 59,63 & 13,88 & 26,11 & 0,38 & 65,82 & 9,96 & 23,93 & 0,28 \\
\hline 0,100 & 58,70 & 9,79 & 30,54 & 0,96 & 70,05 & 7,45 & 21,71 & 0,80 \\
\hline
\end{tabular}

entre 250 e $300 \mathrm{~nm}$.

Na Fig. 4 podemos observar os picos de emissão característicos do aluminato de zinco dopado com o íon $\mathrm{Eu}^{3+}$. $\mathrm{O}$ espectro de luminescência deste íon é significativamente influenciado pela rede cristalina utilizada como hospedeira por causa das transições eletrônicas envolvendo apenas a redistribuição de elétrons dentro da subcamada $4 f$ [5]. Estas transições podem ocorrer via dipolo elétrico forçado (DEF), dipolo magnético (DM) ou quadripolo elétrico (QM) e são provocadas pela ação do campo ligante gerado pela rede hospedeira que distorce o íon livre [13]. O pico de emissão mais intenso centrado em $613 \mathrm{~nm}$, correspondente a transição ${ }^{5} D_{0} \rightarrow{ }^{7} F_{2}$ que ocorre via dipolo elétrico forçado (DEF), possui intensidade consideravelmente superior à do pico centrado em $591 \mathrm{~nm}$, transição ${ }^{5} D_{0} \rightarrow{ }^{7} F_{l}$, que ocorre via

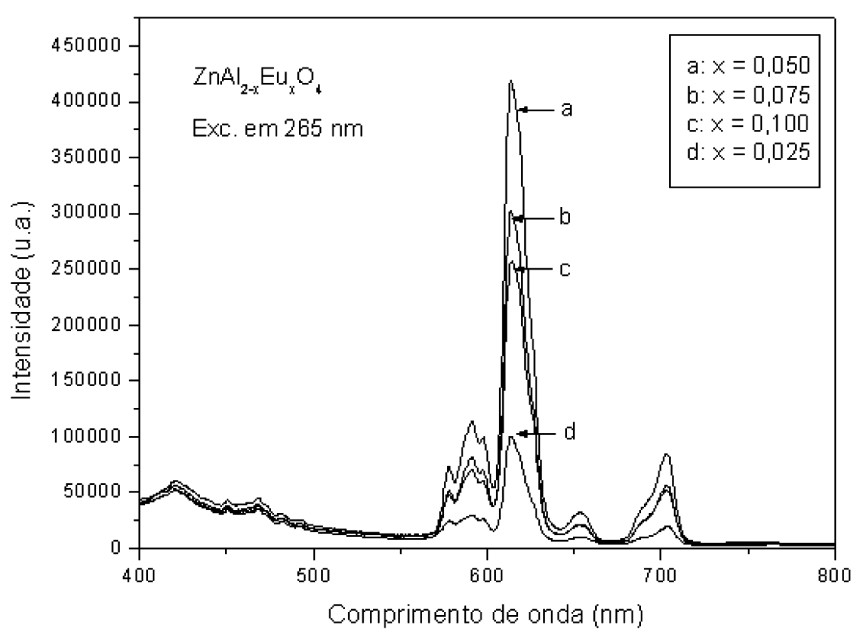

Figura 5: Espectros de emissão para o $\mathrm{ZnAl}_{2} \mathrm{O}_{4}: \mathrm{Eu}^{3+}$ em função da concentração de dopante.

[Figure 5: Emission spectra of ZnAl2O4:Eu ${ }^{3+}$ as a function of the dopant concentration.] dipolo magnético (DM), indicando que o íon $\mathrm{Eu}^{3+}$ encontra-se em uma ambiente de baixa simetria [13]. Os picos de emissão com menor intensidade observados em 578, 653 e $703 \mathrm{~nm}$ correspondem as transições ${ }^{5} D_{0} \rightarrow{ }^{7} F_{0},{ }^{5} D_{0} \rightarrow{ }^{7} F_{3}$ e ${ }^{5} D_{0} \rightarrow{ }^{7} F_{4}$, respectivamente. Os espectros de emissão também apresentam picos de emissão entre 420 e $490 \mathrm{~nm}$, provavelmente gerados pela matriz $\left(\mathrm{ZnAl}_{2} \mathrm{O}_{4}\right)$.

Os espectros de emissão dos pós de aluminato de zinco dopado com $\mathrm{Eu}^{3+}$ em função da concentração de dopante são mostrados na Fig. 5. Estes espectros de emissão mostram uma significativa variação da intensidade relativa de luminescência em função da concentração de dopante, com intensidade máxima alcançada para $0,05 \mathrm{~mol} \mathrm{de} \mathrm{Eu}^{3+}$. A baixa luminescência da amostra dopada com $0,025 \mathrm{~mol}$ de $\mathrm{Eu}^{3+}$ é provavelmente uma conseqüência da formação excessiva de óxido de zinco, indicada pela alta intensidade dos picos característicos de difração de raios $\mathrm{X}$ desta fase (Fig. 2), como também da baixa concentração de dopante usada na síntese $(0,025 \mathrm{~mol})$. Em concentrações superiores a $0,05 \mathrm{~mol}$, temos menores intensidades de luminescência como consequiência da diminuição da distância entre íons dopantes (ativadores) dentro da matriz, provocando o processo de relaxação cruzada, que consiste na transferência de energia entre estes íons. Este tipo de efeito não aparece em baixas concentrações, pois a distancia entre ativadores é grande, de tal modo que a transferência de energia entre os mesmos é intensamente dificultada.

\section{CONCLUSÕES}

Foram preparadas nanopartículas de $\mathrm{ZnAl}_{2} \mathrm{O}_{4}$ dopadas com íons $\mathrm{Eu}^{3+}$ pelo método de síntese por reação de combustão, apresentando boa cristalinidade e tamanhos de cristalito inferiores a $20 \mathrm{~nm}$. Por meio dos difratogramas de raios $\mathrm{X}$ foi identificada a fase $\mathrm{ZnAl}_{2} \mathrm{O}_{4}$ (fase majoritária) e traços das fases secundarias $\mathrm{ZnO}$ e $\mathrm{EuAlO}_{3}$. As análises por 
energia dispersiva (EDS) indicam que todas as fases formadas $\left(\mathrm{ZnAl}_{2} \mathrm{O}_{4}, \mathrm{ZnO}\right.$ e $\left.\mathrm{EuAlO}_{3}\right)$ apresentaram certo teor de íons $\mathrm{Eu}^{3+}$. Na fase majoritária estes íons podem estar adsorvidos na superfície das partículas devido a porosidade do espinélio ou dentro da rede hospedeira, em posições octaédricas normalmente ocupadas pelos íons $\mathrm{Al}^{3+}$. Com base no espectro de excitação, foi possível identificar uma forte banda centrada em torno de $265 \mathrm{~nm}$, gerada pela transferência de carga dos íons $\mathrm{O}^{2-}$ para os íons $\mathrm{Eu}^{3+}$. Os espectros de emissão apresentaram as transições ${ }^{5} D_{0} \rightarrow^{7} F_{j(j=0,1,2,3,4)}$, características do $\mathrm{Eu}^{3+}$ e a concentração critica de dopante foi determinada em 0,05 mol de $\mathrm{Eu}^{3+}$, concentração na qual obteve-se maior intensidade de luminescência.

\section{AGRADECIMENTOS}

Os autores agradecem o apoio financeiro da Rede de Nanotecnologia Molecular e de Interfaces - RENAMI/CNPq e FAPESP.

\section{REFERÊNCIAS}

[1] R. J. Hill, J. R. Craig, G. V. Gibbs, Phys. Chem. Minerals 4 (1979) 317.

[2] S. K. Sampath, J. F. Cordaro, J. Am. Ceram. Soc. 3 (1998) 649.

[3] C. F. M Costa, E Tortella, M. R. Morelli, R. H. G. A Kiminami, J. Mater. Syn. Proc. (2002) 86-92.

[4] T. El-Nabarawy, A. A. Attia, M. N. Alaya, Mater. Lett. 24 (1995) 319.

[5] Z. Lou, J. Hao, Thin Solid Films 450 (2004) 334-340.

[6] W. Strek, P. Derén, A. Bednarkiewicz, M. Zawadzki, J. Wrzyszcz, J. Alloys \& Comp. 300-301 (2000) 456-458.

[7] M. García-Hipólito, C. D. Hernández-Pérez, O. AlvarezFregoso, E. Martínez, J. Guzmán-Mendoza, C. Falcony, Optical Mater. 22 (2003) 345-351.

[8] R. Pandey, J. D. Gale, S. K. Sampath, J. M. Recio, J. Am.
Ceram. Soc. 82, 12 (1999) 3337.

[9] J. Wrzyszcz, M. Zawadzki, A. M. Trzeciak, J. Mol. Cataly. A: Chemical 189, 2 (2002) 203-210.

[10] M. C. Marion, E. Garbowski, M. Priment, J. Chem. Soc., Faraday Trans. 11 (1991) 1795.

[11] M. A. Valenzuela, G. Aguilar, P. Bosch, H. Amendáriz, P. Salas, A. Montoya, Cat. Lett. 15 (1992) 179.

[12] R. Bhargava, R. Gallagher. Phys. Rev. Lett. 72 (1994) 416.

[13] J. E. C. Silva, Geração e controle das cores-luz primárias em materiais vítreos dopados com tríades de lantanídeos, Diss. Mestrado, Programa de Pós-Graduação em Ciências, UFPE, (1997).

[14] T. Mimani, J. Alloys \& Comp. 315 (2001) 123-128.

[15] A. C. F. M. Costa, E. Tortella, M. J. Kaufman, M. R. Morelli, R. H. G. A. Kiminami, J. Mater. Sci. 37 (2002) 1-4. [16] M. Zawadzki, J. Wrzyszcz, W. Strek, D. Hreniak, J. Alloys \& Comp. 323-324 (2001) 279-282.

[17] A. C. F. M. Costa, Síntese por reação de combustão, sinterização e caracterização de ferritas Ni-Zn, Tese de Doutorado em Ciências e Engenharia de Materiais, Departamento de Engenharia de Materiais, Universidade Federal de S. Carlos, S. Carlos (2002).

[18] H. Klug, L. Alexander, X-Ray Powder Procedures (Wiley, New York, 1962) p. 491.

[19] Y. Zhang, Y, G. C. Stangle, Preparacion of fine multicomponent oxide ceramic powder by a combustion synthesis process, J. Mater. Res. 9 (1994) 1997-2004.

[20] A. C. F. M. Costa et al. Síntese e caracterização de pós $\mathrm{Ni}_{0,5} \mathrm{Zn}_{0,5} \mathrm{Fe}_{2} \mathrm{O}_{4}$. Anais do $45^{\circ}$ Congresso Brasileiro de Cerâmica, Florianópolis, SC (2001).

[21] C. Dong, PowderX Report, Institute of Physics, Chinese Academy of Sciences, Beijing (1997).

[22] J. Bamg, M. Abboudi, B. Abrams, P. H. Holloway, J. Luminescence 106 (2004) 177-185.

(Rec. 29/11/2004, Ac. 12/02/2005) 